\title{
Surveillance and epidemiology of surgical site infections after cardiothoracic surgery in The Netherlands, 2002-2007
}

\author{
Judith Manniën, PhD, ${ }^{\mathrm{a}}$ Jan C. Wille, BSc, ${ }^{\mathrm{b}}$ Jaap J. Kloek, MD, ${ }^{\mathrm{c}}$ and Birgit H. B. van Benthem, $\mathrm{PhD}^{\mathrm{a}}$
}

Objective: Surgical site infections after cardiothoracic surgery substantially increase the risk for illness, mortality, and costs. Surveillance of surgical site infections might assist in the prevention of these infections. This study describes the Dutch surveillance methods and results of data collected between 2002 and 2007.

\begin{abstract}
Methods: Three cardiothoracic procedures were included: coronary artery bypass graft procedures, valve surgery, and a combination of coronary artery bypass graft procedures with concomitant valve surgery. The surgical site infections were divided into sternal and harvest-site infections. Postdischarge surveillance of surgical site infections was mandatory for sternal wounds and elective for harvest-site wounds, with a follow-up period of 42 postoperative days. Multivariate logistic regression was used for risk factor analysis of coronary artery bypass grafts, with adjustment for random variation among hospitals.
\end{abstract}

Results: Eight of the 16 Dutch cardiothoracic centers participated and collected data on 4066 procedures and 183 surgical site infections, revealing a surgical site infection rate of $2.4 \%$ for sternal wounds and $3.2 \%$ for harvest sites. Sixty-one percent of all surgical site infections were recorded after discharge. For sternal surgical site infections after coronary artery bypass graft procedures, the significant risk factors were rethoracotomy, diabetes, preoperative length of stay, and obesity; for harvest-site infections, the most relevant risk factor was a long time on extracorporeal circulation. Adjusted surgical site infection rates regarding coronary artery bypass graft procedures varied between hospitals from $0.0 \%$ to $9.7 \%$.

Conclusions: Large differences were found in surgical site infection rates between Dutch hospitals, which indicate room for improvement. The follow-up of patients after hospital discharge reduces underestimation of surgical site infection rates. (J Thorac Cardiovasc Surg 2011;141:899-904)

In The Netherlands, about 16,000 patients undergo cardiothoracic surgery in one of 16 specialized hospitals every year. Surgical-site infections (SSIs) after cardiothoracic procedures substantially increase the risk for mortality, illness, and costs. Hollenbeak and colleagues ${ }^{1}$ reported that a deep sternal infection increased the length of hospital stay by 20 days, led to a 36 times increased risk of dying, and increased costs with $\$ 19,000$. The proportion of patients undergoing cardiothoracic procedures at risk for infection is increasing because of the aging population and the growing number of patients with conditions like obesity or diabetes mellitus. SSIs after cardiothoracic surgery should be prevented where possible. It has been demonstrated that in addition to a meticulous surgical technique,

\footnotetext{
From the Centre for Infectious Disease Control, ${ }^{a}$ National Institute for Public Health and the Environment (RIVM), Bilthoven, The Netherlands; the Dutch Institute for Healthcare Improvement (CBO), ${ }^{\mathrm{b}}$ Utrecht, The Netherlands; and the Department of Cardiothoracic Surgery, ${ }^{\mathrm{c}}$ Academic Medical Center, University of Amsterdam, Amsterdam, The Netherlands.

Disclosures: Authors have nothing to disclose with regard to commercial support.

Received for publication Nov 19, 2009; revisions received July 5, 2010; accepted for publication Sept 2, 2010; available ahead of print Nov 22, 2010.

Address for reprints: Birgit H. B. van Benthem, PhD, National Institute for Public Health and the Environment (RIVM), Centre for Infectious Disease Control, PO Box 1 (pb.75), 3720 BA Bilthoven, The Netherlands (E-mail: birgit.van. benthem@rivm.nl).

0022-5223/\$36.00

Copyright (c) 2011 by The American Association for Thoracic Surgery

doi:10.1016/j.jtcvs.2010.09.047
}

good hygiene measures, working according to infection prevention guidelines, and administration of antimicrobial prophylaxis appropriately, surveillance of SSIs might assist in the prevention of these infections. ${ }^{2-6}$ Important aspects of SSI surveillance are clear infection criteria and a standardized method for tracing infections. For reliable comparison of infection rates between hospitals, the rates should be corrected for differences in risk factors or other disturbing factors.

In The Netherlands, SSI surveillance was set up within the national nosocomial infection surveillance network Preventie van Ziekenhuisinfecties door Surveillance (PREZIES) in 1996. ${ }^{7}$ Because the factors that influence the risk for an SSI after cardiothoracic surgery differ from those influencing SSI risk after other surgical procedures (eg, time on extracorporeal circulation), a separate surveillance protocol was developed for cardiothoracic surgery in 2001. This study describes the surveillance methods and the results of data collected between 2002 and 2007. The degree of participation might indicate the feasibility of the surveillance, how much value hospitals attach to it, or both. Variation between hospitals in SSI incidence might indicate possible room for improvement. Furthermore, the results might reveal possible patient groups at high risk for SSIs after cardiothoracic surgery. Overall, the results can be used for improvement of the surveillance methodology and for specific infection prevention improvement activities in the hospitals. 


\section{Abbreviations and Acronyms}

$\begin{aligned} \text { CABG }= & \text { coronary artery bypass graft } \\ \text { ECC }= & \text { extracorporeal circulation } \\ \text { NNIS } & \text { National Nosocomial Infection } \\ & \text { Surveillance } \\ \text { PDS }= & \text { postdischarge surveillance } \\ \text { PREZIES = } & \text { Preventie van Ziekenhuisinfecties } \\ & \text { door Surveillance } \\ \text { SSI } & \text { surgical site infection }\end{aligned}$

\section{MATERIALS AND METHODS}

In 2002, a pilot study took place regarding the surveillance of SSIs after cardiothoracic surgery in 7 Dutch hospitals. After a few adjustments, the definite protocol was agreed on in 2003. For reliable comparison of infection rates between hospitals, only clearly defined procedures that are regularly performed should be included in surveillance systems. Therefore the Dutch surveillance focused on 3 types of cardiothoracic procedures: coronary artery bypass graft (CABG) procedures, valve surgery, and a combination of CABG procedures and concomitant valve surgery. All patients undergoing the 3 selected types of cardiothoracic procedures must be included in the PREZIES surveillance to avoid selection bias.

When these procedures are performed, the sternum is always opened. With CABG procedures, donor grafts are often harvested from the leg (saphenous vein) or arm (radial artery), which results in another incision that might get infected. In addition, internal thoracic arteries can be used as bypass grafts.

The standardized criteria for an SSI developed by the US Centers for Disease Control and Prevention were applied. SSIs after cardiothoracic surgery were classified as sternal wound infections or harvest-site infections. Sternal infections related to the skin were considered superficial SSIs, and infections of the sternum, mediastinum, or both were considered deep SSIs. Harvest-site infections were classified as superficial and deep SSIs, with deep incisional and organ-space infections combined into deep SSIs.

Based on the international literature and the results of the Dutch pilot study, the following risk factors were included in the final PREZIES protocol: age, sex, insulin-dependent diabetes, obesity (body mass index $\geq 30$ ), preoperative duration of hospitalization, whether the operation was performed on an emergency basis, application of both internal thoracic arteries, use of a heart-lung machine (and, if so, the time on extracorporeal circulation $[\mathrm{ECC}]$ ), lowest body temperature during the operation (nasal measurement), duration of intensive care admission, and rethoracotomy (if not executed because of an infection). In case a heart-lung machine was used, the 75th percentile of ECC time was calculated in minutes per type of procedure by using the current data. The registration of nasal carriage of Staphylococcus aureus and culture results (at most 3 microorganisms per infection) was voluntary. Both for screening and clinical isolates, no distinction is made between methicillin-susceptible $S$ aureus or methicillin-resistant $S$ aureus. The American Society of Anesthesiologists physical status classification, wound contamination class, and duration of the operation (which are included in the American National Nosocomial Infection Surveillance [NNIS] risk index) were not included in the protocol because the predictability of these items for an SSI is poor for CABG procedures. ${ }^{8-10}$

Because many cardiothoracic surgery patients are referred from another hospital, the patients often will return to that hospital shortly after the operation. Therefore many SSIs will develop after patients are discharged from the specialized heart center where the procedure took place. To minimize the risk for missing SSIs, within the PREZIES network, postdischarge surveillance (PDS) of SSIs after cardiothoracic procedures was mandatory for sternal wounds and elective for harvest-site wounds. A follow-up period of 42 postoperative days was assigned mainly for practical reasons because most patients returned to the heart center for control at 6 weeks postoperatively. The recommended method by PREZIES for PDS of sternal wounds was addition of a special registration card to the outpatient medical record on which the surgeon noted clinical symptoms and whether a patient had an SSI according to the definitions; an alternative method was examination of the outpatient medical record after the follow-up period had elapsed. ${ }^{11}$ A prerequisite for this alternative method was that the status of the wound had to be clearly described in the records. For postdischarge follow-up of harvest sites, the advice was to give patients a questionnaire that they should return in case of signs of an SSI or otherwise after 6 weeks. In case PDS was performed, the date of PDS could be registered voluntarily. The date of PDS indicated the most recent date on which information was gathered from the patient (eg, the date of postdischarge check-up or the date of infection). The duration of follow-up was determined by the number of days between the operation and the date of PDS.

Every time a hospital sent in data (on average once a year), it received a feedback report per surgical procedure (CABG, valve surgery, or a combination of these) including infection rates for each type of SSI separately (ie, sternal SSIs and harvest-site SSIs). The report also provided expected SSI rates adjusted for the most relevant risk factor or factors. Furthermore, the feedback report included figures showing the SSI rate for each participating hospital per type of procedure and per type of SSI. Within the participating hospitals, feedback reports were usually spread among and discussed with the infection control committee, physicians, managers, and other involved staff.

Within the Dutch PREZIES network, participation is voluntary, and hospital-specific data are kept confidential. Validation visits by a PREZIES team member to each participating hospital take place every 3 years and provide evidence for the reliability and accuracy of the surveillance data. ${ }^{12}$ The process of surveillance, including the quality of the method of data collection, is validated by means of a structured interview. The use of SSI criteria is validated by review of 25 medical records, with the judgment of the validation team as the criterion standard. Yearly workshops for participants are organized by the PREZIES network to provide information, exchange experiences and tips and possible pitfalls regarding the surveillance, discuss possible infection prevention strategies, and practice cases studies.

Multivariate risk factor analysis was only performed for CABG procedures because of the small number of recorded SSIs after valve surgery and after a combination of CABG with concomitant valve surgery. The analyses were executed separately for harvest-site and sternal SSIs, but superficial and deep SSIs were combined. Variables with a $P$ value of less than .2 for their univariate association with SSI were candidates for multivariable logistic regression analysis. In the present multicenter study, patients were clustered by hospital. This level of hierarchy can introduce additional sources of variability and correlation (eg, by hospital-specific treatment policies or risk factors). Therefore a random coefficient model was used to adjust the risk estimates for random variation among hospitals. Manually forward selection was performed by using the likelihood ratio test. Associations between SSIs and exposure to risk factors were estimated by odds ratios and $95 \%$ confidence intervals.

A Kaplan-Meier survival curve was created to present the duration of follow-up of all patients.

All analyses were performed with SAS for Windows software (SAS 9.1; SAS Institute, Inc, Cary, NC).

\section{RESULTS}

Seven of the 16 Dutch specialized cardiothoracic centers participated in the PREZIES pilot surveillance on SSIs in 2002. Afterward, 3 of these hospitals plus 1 additional 
hospital participated using the final protocol. Because the protocol of the pilot study only slightly differed from the final protocol, all data from 2002 to 2007 were included to increase the reliability. Between 2002 and 2007, 8 hospitals collected data on 4066 patients undergoing CABG surgery, valve surgery, or a combination of CABG surgery with concomitant valve surgery.

Table 1 describes the characteristics of the patient population. The number of procedures per hospital varied from 108 to 1146 . The data revealed some remarkable differences in patient and procedure characteristics between hospitals. For example, the percentage of CABG procedures in which a heart-lung machine was used varied between $68 \%$ and $100 \%$, and if used, the median ECC time varied between 64 and 123 minutes. Bilateral thoracic arteries were used in $0 \%$ to $16 \%$ of $\mathrm{CABG}$ procedures, with an outlier of $43 \%$. In CABG procedures, the percentage of patients with a body mass index of $30 \mathrm{~kg} / \mathrm{m}^{2}$ or greater varied from $18.5 \%$ to $28 \%$. Overall, the median lowest body temperature varied between $30^{\circ} \mathrm{C}$ and $35^{\circ} \mathrm{C}$ among the hospitals.

In total, 4066 surgical procedures and 183 SSIs were registered. Fifty-three deep sternal SSIs (cumulative incidence, $1.3 \%$ ) and 44 superficial sternal SSIs (cumulative incidence, $1.1 \%$ ) were included. Of the 2691 patients with a harvest site, 86 had an SSI at the harvest site (cumulative incidence, $3.2 \%$ ), of which 13 were deep SSIs. The cumulative incidences of SSIs per type of procedure are shown in Table 2. Six patients had both a sternal and a harvest-site SSI.

The variation in SSI incidence between hospitals was larger for harvest-site SSIs than for sternal SSIs. This might reflect the fact that for harvest sites, PDS was voluntary, and

TABLE 1. Characteristics of patients undergoing cardiothoracic procedures

\begin{tabular}{lrrrc}
\hline & & CABG & Valve & CABG/valve \\
& Overall & surgery & surgery & surgery \\
\hline No. of procedures & 4066 & 2732 & 808 & 526 \\
Male sex (\%) & 71 & 76 & 56 & 69 \\
Median age (y) & 67 & 67 & 66 & 71 \\
Emergency procedure (\%) & 7 & 7 & 6 & 4 \\
Preoperative LOS $\geq 2 \mathrm{~d}(\%)$ & 33 & 35 & 27 & 31 \\
Body mass index & 20 & 21 & 16 & 17 \\
$\quad \geq 30 \mathrm{~kg} / \mathrm{m}^{2}(\%)$ & & & & \\
Diabetes (\%) & 7 & 9 & 3 & 6 \\
Lowest body temperature & 25 & 27 & 21 & 20 \\
$\quad>34^{\circ} \mathrm{C}(\%)$ & & & & \\
ICU LOS $\geq 2 \mathrm{~d}(\%)$ & 49 & 44 & 57 & 74 \\
Rethoracotomy (\%) & 9 & 7 & 13 & 14 \\
Heart-lung machine (\%) & 92 & 88 & 100 & 100 \\
Median duration of & 109 & 99 & 123 & 165 \\
$\quad$ perfusion (min) & & & & \\
Use of bilateral thoracic & 6 & 9 & 0 & 3 \\
$\quad$ arteries (\%) & & & & \\
\hline$C A B G$, Coronary artery bypass graft; $L O S$, length of stay; ICU, intensive care unit.
\end{tabular}

3 hospitals did not perform active PDS for these wounds. Consequently, these 3 hospitals recorded the lowest SSI incidences for harvest sites. For CABG procedures, the sternal SSI incidence varied from $0.0 \%$ to $4.5 \%$ between hospitals, and the SSI incidence for harvest sites ranged from $0.4 \%$ to $6.9 \%$ between hospitals.

For patients without an SSI, the median length of stay was 10 days: 9 days for CABG surgery, 10 days for valve surgery, and 12 days for a combination of CABG and valve surgery. Regarding CABG surgery, the median duration of admission for patients with a superficial sternal SSI was 12 days, that for patients with a deep sternal SSI was 19 days, and that for patients with a harvest-site SSI was 10 days. Thus there was a median increase of 3, 10, and 1 days, respectively, compared with patients without an SSI after CABG surgery. PDS led to the reporting of 24 deep sternal SSIs (45\% of 53), 24 superficial sternal SSIs (55\% of 44$)$, and 64 harvest-site SSIs (74\% of 86$)$ after the patients had left the heart centers. Table 3 shows the number of SSIs detected during admission and after discharge specifically for $\mathrm{CABG}$ procedures.

Figure 1 shows the Kaplan-Meier survival curve of sternal and harvest-site SSIs after the included cardiothoracic procedures. Regarding the 3889 patients without an SSI, the date of PDS was missing for 801 patients. In these cases, the duration of follow-up was determined as the period from surgical intervention to discharge. Seventy-three percent (2836) of the 3889 patients without an SSI were followed for at least 42 postoperative days, which was the surveillance period according to the protocol. The risk for harvest-site SSIs seemed to be steady during the first 4 postoperative weeks. The risk for sternal SSIs was highest between days 6 and 16 postoperatively. The number of days between surgical intervention and infection hardly differed between deep and superficial sternal SSIs.

Multilevel multivariate risk factor analysis was performed for CABG procedures (Table 4). For sternal SSIs, the significant risk factors were rethoracotomy (not executed because of an infection), diabetes, preoperative length of stay, and obesity. For harvest-site SSIs, the most relevant risk factor appeared to be an ECC time of longer than the 75th percentile for $\mathrm{CABG}$ procedures, which was 123 minutes.

A culture result was available for $46(87 \%)$ of the 53 deep sternal SSIs and for $26(59 \%)$ of the 44 superficial SSIs. Fifty-eight percent (49/84) of the isolated microorganisms were identified as staphylococci (37 being S aureus). For 41 (48\%) of the 86 harvest-site SSIs, a culture result was recorded. Thirty-one percent (19/61) of the isolated microorganisms were identified as staphylococci (15 being $S$ aureus). Of the 514 patients with a positive nose swab result for $S$ aureus, $31(6.0 \%)$ had an SSI. Of the 1350 patients with a negative nose swab result for $S$ aureus, $53(3.9 \%)$ had an SSI. $S$ aureus was isolated $11(21 \%)$ times $(P=.07)$. 
TABLE 2. Cumulative incidence of SSIs per type of procedure

\begin{tabular}{|c|c|c|c|c|c|c|}
\hline & \multicolumn{2}{|c|}{ CABG surgery } & \multicolumn{2}{|r|}{ Valve surgery } & \multicolumn{2}{|c|}{ CABG/valve surgery } \\
\hline & No. & $\begin{array}{c}\text { Cumulative incidence } \\
(\mathbf{9 5} \% \mathrm{CI})\end{array}$ & No. & $\begin{array}{c}\text { Cumulative incidence } \\
(\mathbf{9 5} \% \mathbf{C I})\end{array}$ & No. & $\begin{array}{c}\text { Cumulative incidence } \\
(95 \% \text { CI })\end{array}$ \\
\hline No. of procedures & 2732 & & 808 & & 526 & \\
\hline Sternal SSIs & 66 & $2.4(1.9-3.1)$ & 14 & $1.7(1.0-2.9)$ & 17 & $3.2(2.0-5.1)$ \\
\hline Deep sternal SSIs & 33 & $1.2(0.9-1.7)$ & 11 & $1.4(0.8-2.4)$ & 9 & $1.7(0.9-3.2)$ \\
\hline Superficial sternal SSIs & 33 & $1.2(0.9-1.7)$ & 3 & $0.4(0.1-1.1)$ & 8 & $1.5(0.8-3.0)$ \\
\hline No. of harvest sites & 2289 & & & & 402 & \\
\hline Harvest-site SSIs & 67 & $2.9(2.3-3.7)$ & & & 19 & $4.7(3.0-7.3)$ \\
\hline
\end{tabular}

SSI, Surgical site infection; $C A B G$, coronary artery bypass graft; $C I$, confidence interval.

\section{DISCUSSION}

Advantages of the Dutch surveillance are that specific risk factors for SSIs after cardiothoracic surgery were included, PDS was mandatory for sternal SSIs, and the execution of the surveillance (including the application of SSI criteria) was validated in all participating hospitals every 3 years. The extra risk factors seemed relevant, as shown by means of multivariate logistic regression analyses, such as rethoracotomy, obesity, diabetes, and ECC time, which are not included in the regular SSI surveillance in The Netherlands. Focusing surveillance on a small number of indicator procedures will save time and allow registration of procedure-specific relevant risk factors. PDS was important because $58 \%$ of the registered sternal SSIs and $79 \%$ of the registered harvest-site SSIs developed after the patient had left the hospital where the operation took place.

A surveillance period of 42 postoperative days was chosen because most patients return to the heart center for control at 6 weeks postoperatively. However, the American SSI surveillance methods, developed by the Centers for Disease Control and Prevention, are accepted and copied by many countries worldwide. Therefore we decided to adjust our protocol from January 2009 and changed the surveillance period to 30 days postoperatively for harvest-site SSIs and superficial sternal SSIs and 1 year postoperatively for deep sternal SSIs. Shortening the surveillance period from 42 to 30 days for harvest-site SSIs and superficial sternal SSIs will decrease the number of recorded SSIs. In the cur-

TABLE 3. Number of SSIs detected during admission or after discharge for CABG procedures

\begin{tabular}{llllll}
\hline & \multicolumn{2}{c}{ During admission } & & \multicolumn{2}{c}{ After discharge } \\
\cline { 2 - 3 } \cline { 5 - 6 } Type of SSI & No. & $\%$ & & No. & $\%$ \\
\hline Sternal SSI & & & & \\
$\quad$ Overall & 28 & 42 & & 38 & 58 \\
$\quad$ Deep & 15 & 45 & & 18 & 55 \\
$\quad$ Superficial & 13 & 39 & & 20 & 61 \\
Harvest-site SSI & & & & \\
$\quad$ Overall & 14 & 21 & & 53 & 79 \\
\hline
\end{tabular}

$S S I$, Surgical site infection; $C A B G$, coronary artery bypass graft. rent study, $13 \%$ of recorded SSIs developed between 30 and 42 days postoperatively.

Our study showed for all included cardiothoracic procedures a cumulative SSI incidence of $2.4 \%$ for sternal SSIs and $3.2 \%$ for harvest-site SSIs. For CABG procedures only, the cumulative SSI incidence was $2.4 \%$ for sternal SSIs and $2.9 \%$ for harvest-site SSIs. Large differences were found between Dutch hospitals, which indicate that there is room for improvement.

Results from other studies reveal a wide range of SSI incidences after cardiothoracic procedures. However, comparisons should be made with caution for several reasons: different SSI criteria, different duration of follow-up, different intensity of PDS, and different types of cardiothoracic procedures. Because of these pitfalls and because the primary aim of a national surveillance system is to decrease the SSI incidence within a country, the focus should be on following time trends within a country instead of comparing SSI incidences between countries. However, a decrease in the SSI incidence might be precluded because the aging population and the growing number of patients with conditions like obesity or diabetes mellitus probably increase the infection risk.

In the current study, rethoracotomy (not executed because of an infection), diabetes, preoperative length of stay, and

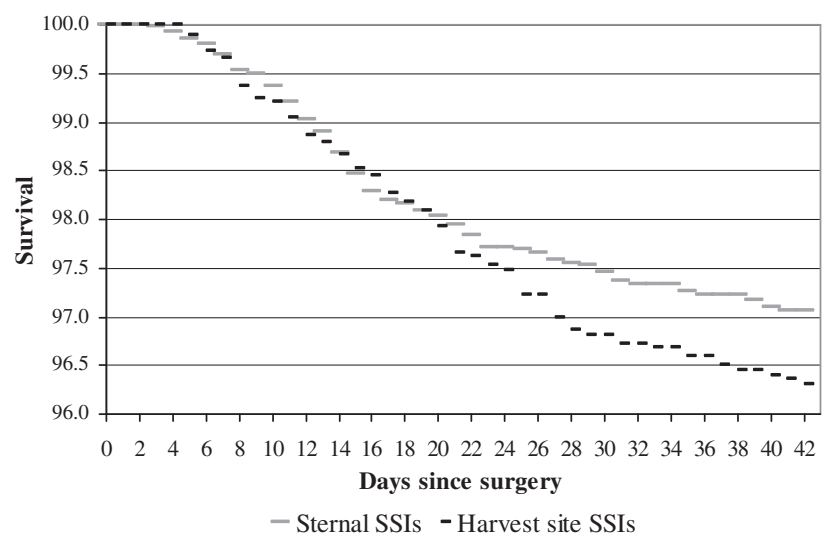

FIGURE 1. Kaplan-Meier survival curve of sternal and harvest-site SSIs after cardiothoracic surgery. 
TABLE 4. Multilevel multivariate risk factor analyses for CABG procedures

\begin{tabular}{llccc}
\hline \multirow{2}{*}{ Type of SSI } & \multicolumn{1}{c}{ Risk factor } & $\begin{array}{c}\text { Odds } \\
\text { ratio }\end{array}$ & $\mathbf{9 5 \%}$ CI & $\begin{array}{c}\boldsymbol{P} \\
\text { value }\end{array}$ \\
\hline Sternal SSIs & Rethoracotomy & 5.2 & $2.5-10.6$ & .001 \\
& Diabetes & 2.5 & $1.1-5.5$ & .03 \\
& Preoperative LOS $>3 \mathrm{~d}$ & 2.1 & $1.1-4.1$ & .04 \\
& Obesity & 2.0 & $1.0-3.8$ & .04 \\
Harvest-site SSIs & ECC time $>123 \mathrm{~min}$ & 1.8 & $0.9-3.7$ & .08 \\
\hline
\end{tabular}

Based on 2732 procedures for sternal SSIs and 2289 procedures for harvest-site SSIs. $C A B G$, Coronary artery bypass graft; $S S I$, surgical site infection; $C I$, confidence interval; $L O S$, length of stay; $E C C$, time on extracorporeal circulation (75th percentile $=123$ minutes).

obesity were related to the development of sternal SSIs, and a long ECC time was related to the development of harvestsite SSIs. Preoperative length of stay is probably a marker for comorbidity. Some other studies found similar risk factors, ${ }^{13,14}$ and others showed many other risk factors that are not recorded within PREZIES (current cigarette smoking, chronic obstructive pulmonary disease, renal insufficiency, and postoperative bleeding) ${ }^{8,15-18}$ However, to keep the surveillance feasible to many hospitals, the amount of data to be collected should be limited. PREZIES focuses on nonimpressionable factors for benchmarking purposes. From 2009, as part of the safety program "Prevent injury, work safe in Dutch hospitals" (2008-2012), a bundle of 4 optional process measures was added to the protocol, including antimicrobial prophylaxis (agent and timing), preoperative hair removal, normothermia, and frequency of opening doors in the operating room. In addition, the EUROScore was added to the protocol as a mandatory item. The EUROScore includes 9 patient-related factors (eg, chronic pulmonary disease, neurologic dysfunction, and previous cardiac surgery), 4 cardiac-related factors (eg, recent myocardial infarction and pulmonary hypertension), and 4 operation-related factors (eg, postinfarct septal rupture). The EUROScore was originally developed to predict mortality risk but has been shown to also predict SSI risk. ${ }^{19}$ Other studies often use the NNIS risk index. However, the items of this index (wound contamination class, duration of operation, and American Society of Anesthesiologists classification) are not strongly related to SSI risk (harvest-site and sternal SSIs combined) after cardiothoracic surgery. ${ }^{8}$ In the present study, no significant risk factors were identified for harvest-site infections. For these infections, the NNIS risk index might be relevant after all. ${ }^{9,10,20}$

Repeatedly, studies have demonstrated that surveillance contributes to the reduction of SSIs. ${ }^{2,6}$ In The Netherlands, the usefulness of SSI surveillance is endorsed by the Dutch authorities. For instance, the Dutch Healthcare Inspectorate included SSI surveillance as a hospitalwide structure indicator in the basic set of Hospital Performance Indicators from 2004, and in 2008 also strongly recommended all cardiothoracic centers in The
Netherlands to participate in the PREZIES module regarding SSIs after cardiothoracic surgery. Furthermore, recognition is revealed by inclusion of the cardiothoracic procedures as indicator procedures within the national safety program "Prevent injury, work safe in Dutch hospitals" (2008-2012), which was developed by the Ministry of Health, the Healthcare Inspectorate, the Dutch Hospitals Association, and others and aims to reach an SSI rate of less than the 25th percentile of the data collected between 2002 and 2007. Between 2004 and 2008, each year only 2 or 3 hospitals participated in the SSI surveillance after cardiothoracic procedures of the PREZIES network. The above-mentioned efforts have resulted in 6 of the 16 Dutch cardiothoracic centers participating in the current surveillance module in 2009. In addition, publication of variation in SSI rates among hospitals, as done in this study, indicates room for improvement and might further increase the awareness in hospitals of the preventable burden and the usefulness of surveillance and, with that, the number of hospitals willing to participate. Although most of the significant risk factors in multivariate analysis are a marker of the patient's health status, decreasing the number of rethoracotomies and the ECC time, where possible, will decrease the incidence of SSIs.

We thank all the infection control practitioners, medical specialists, and nurses at the participating hospitals for their invaluable contribution to the collection of data.

\section{References}

1. Hollenbeak CS, Murphy DM, Koenig S, Woodward RS, Dunagan WC, Fraser VJ. The clinical and economic impact of deep chest surgical site infections following coronary artery bypass graft surgery. Chest. 2000;118: $397-402$.

2. Gaynes R, Richards C, Edwards J, et al. Feeding back surveillance data to prevent hospital-acquired infections. Emerg Infect Dis. 2001;7:295-8.

3. Geubbels EL, Nagelkerke NJ, Mintjes-De Groot AJ, Vandenbroucke-Grauls CM, Grobbee DE, De Boer AS. Reduced risk of surgical site infections through surveillance in a network. Int J Qual Health Care. 2006;18:127-33.

4. Haley RW, Culver DH, White JW, et al. The efficacy of infection surveillance and control programs in preventing nosocomial infections in US hospitals. Am J Epidemiol. 1985;121:182-205

5. Harbarth S, Sax H, Gastmeier P. The preventable proportion of nosocomial infections: an overview of published reports. J Hosp Infect. 2003;54:258-66.

6. Mannien J, van den Hof S, Muilwijk J, van den Broek PJ, van Benthem B, Wille JC. Trends in the incidence of surgical site infection in the Netherlands. Infect Control Hosp Epidemiol. 2008;29:1132-8.

7. Geubbels EL, Mintjes-de Groot AJ, van den Berg JM, de Boer AS. An operating surveillance system of surgical site infections in The Netherlands: results of the PREZIES national surveillance network. Preventie van Ziekenhuisinfecties door Surveillance. Infect Control Hosp Epidemiol. 2000;21:311-8.

8. Batista R, Kaye K, Yokoe DS. Admission-specific chronic disease scores as alternative predictors of surgical site infection for patients undergoing coronary artery bypass graft surgery. Infect Control Hosp Epidemiol. 2006;27:802-8

9. Bundy JK, Gonzalez VR, Barnard BM, Hardy RJ, DuPont HL. Gender risk differences for surgical site infections among a primary coronary artery bypass graft surgery cohort: 1995-1998. Am J Infect Control. 2006;34:114-21.

10. Friedman ND, Bull AL, Russo PL, Gurrin L, Richards M. Performance of the national nosocomial infections surveillance risk index in predicting surgical site infection in Australia. Infect Control Hosp Epidemiol. 2007;28: $55-9$. 
11. Manniën J, Wille JC, Snoeren RL, van den Hof S. Impact of postdischarge surveillance on surgical site infection rates for several surgical procedures: results from the nosocomial surveillance network in the Netherlands. Infect Control Hosp Epidemiol. 2006;27:809-16.

12. Manniën J, van der Zeeuw AE, Wille JC, van den Hof S. Validation of surgical site infection surveillance in The Netherlands. Infect Control Hosp Epidemiol. 2007;28:36-41.

13. Lindhout AH, Wouters CW, Noyez L. Influence of obesity on in-hospital and early mortality and morbidity after myocardial revascularization. Eur J Cardiothorac Surg. 2004;26:535-41.

14. Talbot TR. Diabetes mellitus and cardiothoracic surgical site infections. Am J Infect Control. 2005;33:353-9.

15. Birkmeyer NJ, Charlesworth DC, Hernandez F, et al. Obesity and risk of adverse outcomes associated with coronary artery bypass surgery. Northern New England Cardiovascular Disease Study Group. Circulation. 1998;97:1689-94.
16. Cayci C, Russo M, Cheema F, et al. Risk analysis of deep sternal wound infections and their impact on long-term survival: a propensity analysis. Ann Plast Surg. 2008;61:294-301.

17. Furnary AP, Gao G, Grunkemeier GL, et al. Continuous insulin infusion reduces mortality in patients with diabetes undergoing coronary artery bypass grafting. J Thorac Cardiovasc Surg. 2003;125:1007-21.

18. Segers P, de Jong AP, Kloek JJ, Spanjaard L, de Mol BA. Risk control of surgical site infection after cardiothoracic surgery. J Hosp Infect. 2006;62: 437-45.

19. Paul M, Raz A, Leibovici L, Madar H, Holinger R, Rubinovitch B. Sternal wound infection after coronary artery bypass graft surgery: validation of existing risk scores. J Thorac Cardiovasc Surg. 2007;133:397-403.

20. Lepelletier D, Perron S, Bizouarn P, et al. Surgical site infection after cardiac surgery: incidence, microbiology, and risk factors. Infect Control Hosp Epidemiol. 2005;26:466-72. 